\title{
Salud mental en población víctima del conflicto armado en Colombia
}

\section{Mental health in population victims of the armed conflict in Colombia}

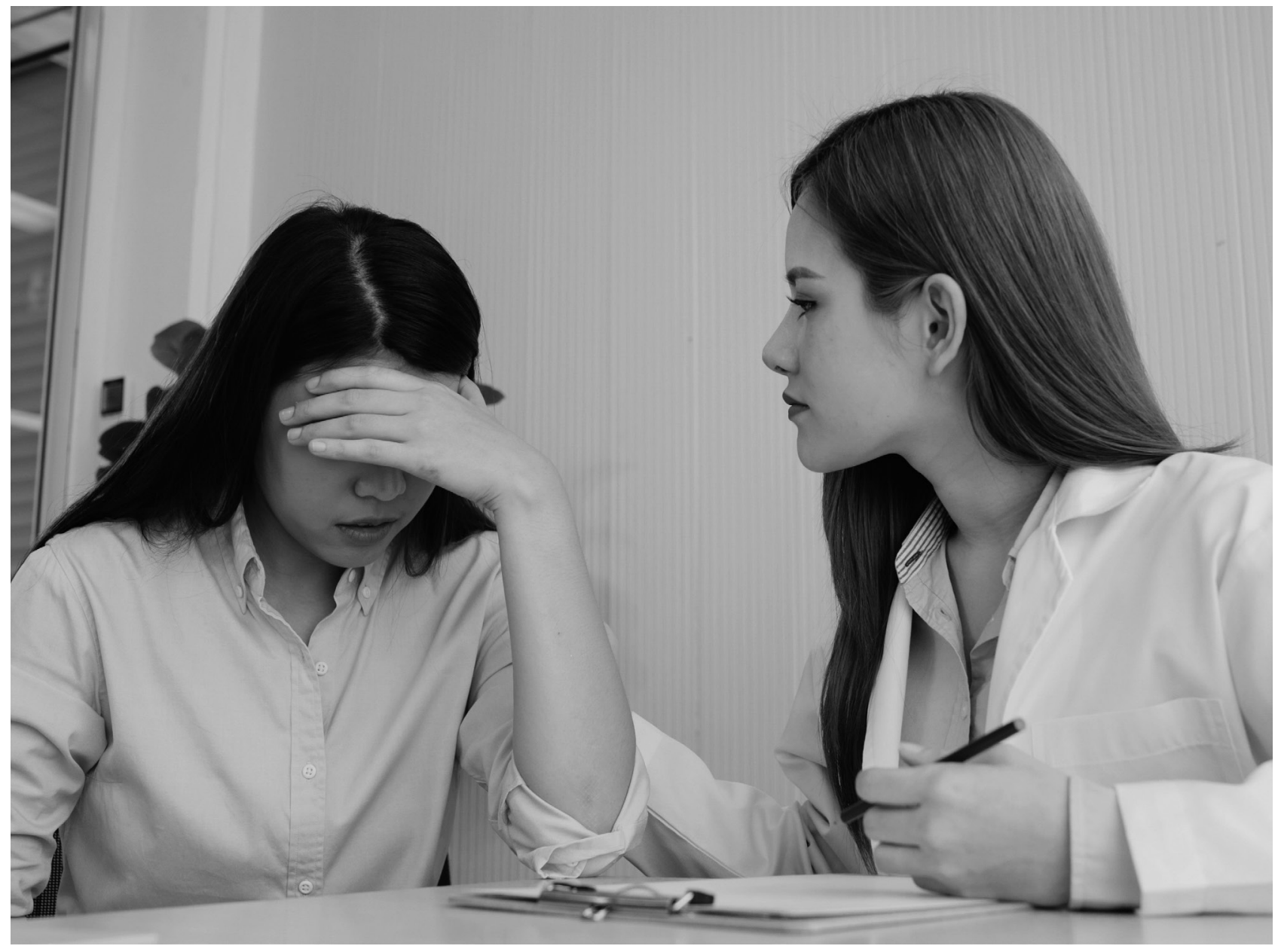




\title{
Salud mental en población víctima del conflicto armado en Colombia ${ }^{1}$ \\ Mental health in population victims of the armed conflict in Colombia
}

\author{
Juan Gabriel Castañeda Polanco, ${ }^{2}$ José Antonio Camargo Barrero ${ }^{3}$
}

Artículo recibido en agosto de 2017; artículo aceptado en febrero de 2018.

Este artículo puede compartirse bajo la Licencia Creative Commons Atribución-No Comercial-Compartir lgual 2.0 Genérica y se referencia usando el siguiente formato: Castañeda, J. \& Camargo, J. (2018). Salud mental en población víctima del conflicto armado en Colombia. I+D Revista de Investigaciones, 12 (2), 19-26. DOI: https://doi.org/10.33304/revinv.v12n2-2018002

\begin{abstract}
Resumen
La presente investigación describió la salud mental en población víctima del conflicto armado colombiano. El estudio fue de tipo cuantitativo descriptivo, con un diseño no experimental. La muestra final estuvo constituida por 265 personas; se les aplicó una ficha de datos sociodemográficos y el cuestionario GHQ-12. Los resultados muestran que el $37 \%$ de la población presenta una baja puntuación en su bienestar psicológico y un $77 \%$ bajos niveles en su función social. Los resultados sugieren establecer correlaciones con tipos de hechos victimizantes como el desplazamiento forzado, asimismo, realizar exámenes más específicos para discriminar diferentes problemas de salud mental relacionados con trastornos y comportamiento disfuncional en la población.
\end{abstract}

Palabras clave: salud mental, GHQ-12, conflicto armado, víctimas.

\begin{abstract}
The present investigation described Mental health in the population victim of the Colombian armed conflict. The study was of quantitative descriptive type, with a non-experimental design. The final sample consisted of 265 people; A sociodemographic data sheet and the GHQ-12 Questionnaire were applied. The results show that 37\% of the population presents a low score in their psychological well-being and $77 \%$ low levels in their social function. The results suggest correlations with types of victimizing events such as forced displacement, as well as conducting more specific Mental Health examinations to discriminate among the population between different mental health problems that are related to disorders and dysfunctional behavior in the population.
\end{abstract}

Keywords: mental health, GHQ-12, armed conflict, victims.

1. Tipo de artículo empírico de enfoque cuantitativo, resultado de un proyecto de investigación en curso, perteneciente al área de psicología, desarrollado en el grupo de investigación Desarrollo Regional MD, fue financiado por la Corporación Universitaria Minuto de Dios - Uniminuto de la ciudad de Bogotá (Colombia). Dirección: Calle 57 n. 17 - 47, PBX:2916520, ext. 13276. Fecha de inicio: 1 febrero de 2017, fecha de terminación: 30 noviembre de 2017.

2. Psicólogo, Universidad Católica de Colombia. Magister en Psicología, Universidad Católica de Colombia. Docente- investigador del grupo Desarrollo Regional MD (Colombia): Corporación Universitaria Minuto de Dios - Uniminuto de la ciudad de Bogotá (Colombia). Dirección: Calle 57 n. 17 - 47 , PBX 2916520, ext. 13276. ORCID ID: https://orcid.org/0000-0001-7632-7526. Correo electrónico institucional: jucastaneda@uniminuto.edu.

3. Psicólogo, Fundación Universitaria del Espinal. Maestría Intervención social en las sociedades del conocimiento, UNIR. Docente- investigador del grupo Desarrollo Regional MD. (Colombia): Corporación Universitaria Minuto de Dios - Uniminuto de la ciudad de Bogotá (Colombia). Dirección: Calle 57 n. 17 47, PBX 2916520 ext. 13276. ORCID ID: https://orcid.org/0000-0003-3175-71109. Correo electrónico institucional: jose.camargo@uniminuto.edu. 


\section{Introducción}

Los conflictos prolongados, como afirman López et al. (2013), Cortés et al. (2016), suelen afectar la infraestructura física, la economía, las instituciones, a las personas en el ámbito psicológico y a la sociedad en general. Las consecuencias de tal destrucción suelen manifestarse durante los posconflictos, requiriéndose recursos para reconstruir lo destruido. En cuanto al tejido social y psicológico, la reconstrucción es más compleja y requiere además inversiones de largo aliento para investigar las consecuencias psicológicas y sociales que ha dejado el conflicto, como es el caso de Colombia (Peña, Martínez \& Leal, 2013).

El problema es más complejo cuando se relacionan las condiciones de victimización del conflicto armado con la vulneración de los derechos humanos, situación que ha sido ampliamente discutida, argumentada y respaldada por autores como Sen $(2000,2009)$ y Fukuda (2012), quienes afirman que el derecho al desarrollo que asegure unas condiciones básicas de nutrición, salud, educación, equidad, justicia y participación política es un derecho que favorece la calidad de vida, que en el caso de las víctimas del conflicto armado es fundamental para la reparación de los derechos vulnerados.

En cuanto a la salud mental, ésta es definida por la Organización Mundial de la Salud [OMS] (2007), como"un estado de bienestar en el cual el individuo es consciente de sus propias capacidades, puede afrontar las tensiones normales de la vida, puede trabajar de forma productiva y fructífera y es capaz de hacer una contribución a su comunidad".

Otras posturas definen la salud mental como un estado subjetivo de bienestar caracterizado por una serie de factores que engloban las dimensiones físicas, culturales, psicosociales, económicas y espirituales de la persona, que se convierten en el sustento de su existencia, lo cual amplía el constructo de salud mental y no lo limita a la simple ausencia de enfermedad (Marks, Murray, Evans \& Willing, 2008; Moreno \& Moriana, 2012; Oblitas, 2009).

El concepto de salud mental es importante ya que éste integra todo un "(...) sistema complejo en el que están interrelacionados aspectos de orden subjetivo y objetivo que se dimensionan desde factores sociales, culturales, biológicos y cognitivos" (Núñez, et al., 2010). Desde esta perspectiva, se puede afirmar que este constructo posee aspectos objetivos y subjetivo que confluyen en la valoración de aquellos factores asociados a calidad de vida (Meeberg, 1993; Cambell, 1981, citado por Urzúa y Caqueo, 2012).
Por otro lado, existen diferentes concepciones sobre la salud mental, las cuales dependen del marco epistemológico de referencia del cual se desprenden y la óptica desde la cual se aborde, siendo la más utilizada la perspectiva biomédica, la cual se centra en los síntomas identificables a través del Manual Diagnóstico y Estadístico de los Trastornos Mentales (DSM V) y la Clasificación Estadística Internacional de Enfermedades y Problemas Relacionados con la Salud (CIE 10). Otras posturas, como las conductuales y cognitivas, valoran más la adaptabilidad del sujeto a su entorno como referente de estabilidad mental, mientras que desde un ángulo socioeconómico se concibe la salud mental como un derecho personal y de desarrollo social (Jaramillo \& Restrepo, 2012).

Por tanto, y teniendo en cuenta el significado y características objetivas y subjetivas de la salud mental, la relación existente entre esta y el conflicto armado en Colombia puede entenderse como un desafío para las autoridades sanitarias y para la comunidad en general, ya que es una variable que requiere ser estudiada con la población involucrada en el conflicto (Pizarro, 2005, citado por Jiménez, 2009).

El objetivo de la presente investigación fue describir las características de salud mental de las víctimas del conflicto armado colombiano que participaron en el estudio, por medio del cuestionario de Salud General GHQ-12.

\section{Método}

\section{Tipo de estudio}

Este estudio es de tipo cuantitativo, descriptivo y transversal, con un diseño no experimental.

\section{Participantes}

La muestra fue no probabilística intencional y estuvo constituida por 265 víctimas directas del conflicto armado interno colombiano identificadas por la Unidad de Atención y Orientación (UAO), en el municipio de Soacha. Las edades oscilaron en un rango de 18 a 60 años, de los cuales 191 (72,1\%) fueron mujeres y el restante $27,9 \%, 74$ hombres.

\section{Materiales e instrumentos}

\section{Ficha de datos sociodemográficos}

Se creó un cuestionario para determinar el tipo de hecho violento del que fueron víctimas. La muestra del estudio valoraba antecedentes sociodemográficos como edad, 
nivel educativo, estado civil, etc.

\section{Cuestionario de Salud General de Goldberg GHQ-12.}

Instrumento de cribado de problemas psicológicos y sociales en población general y clínica. Está constituido por 12 ítems, de los cuales 6 son afirmaciones negativas y 6 positivas. Las preguntas están diseñadas en escala Likert, con 4 puntos por pregunta, con puntuación total del instrumento de 0 a 36 y con una consistencia interna de Alfas de Cronbach de 0,82 y 0,86 (Villa, Zuluaga \& Restrepo, 2013).

\section{Análisis estadístico}

El procesamiento y análisis de los datos se realizó mediante el programa estadístico Statistical Package for Social Science (SPSS). Los resultados se presentan mediante frecuencias, medias y porcentajes de las variables cuantitativas trabajadas.

\section{Procedimiento}

El proyecto fue aprobado por el Comité de ética de la Corporación Universitaria Minuto de Dios, Centro Regional Soacha.

Previo consentimiento informado y explicación de los objetivos de la investigación, se prosiguió a la aplicación de los dos instrumentos, la ficha de datos sociodemográficos y el cuestionario GHQ-12 a los participantes de la Unidad de Atención y Orientación (UAO) de víctimas del municipio de Soacha. Los cuestionarios fueron autodiligenciados con ayuda del entrevistador, y la duración de la aplicación fue en promedio de quince minutos.

\section{Resultados}

Los resultados dejan en evidencia que el estado civil más frecuente en la muestra (Tabla 1) es el soltero, con un $34,7 \%$, seguido de unión libre, con $33,2 \%$ y casados $16,2 \%$. El 15,9\% restante está distribuido entre las otras opciones, siendo divorciados el de menor frecuencia, con un 1,9\%. En la Tabla 2 se presentan las frecuencias relacionadas

Tabla 1

\section{Datos sociodemográficos de la muestra}

\begin{tabular}{lccc}
\hline & & Frecuencia & Porcentaje \\
\hline \multirow{2}{*}{ Sexo } & Hombre & 74 & $27,9 \%$ \\
& Mujer & 191 & $72,1 \%$ \\
& Soltero & 92 & $34,7 \%$ \\
& Casado & 43 & $16,2 \%$
\end{tabular}

\begin{tabular}{cccc} 
& Divorciado & 5 & $1,9 \%$ \\
Estado civil & Separado & 17 & $6,4 \%$ \\
& Viudo & 20 & $7,5 \%$ \\
& Unión libre & 88 & $33,2 \%$ \\
\hline \multirow{3}{*}{ Nivel educativo } & Ninguno & 15 & $5,7 \%$ \\
& Primaria & 117 & $44,2 \%$ \\
& Secundaria & 106 & $40,0 \%$ \\
& Técnico -tecnológico & 24 & $9,1 \%$ \\
& Universitario & 3 & $1,1 \%$ \\
\hline
\end{tabular}

Fuente: Autores

con los hechos victimizantes en la población objeto del estudio, entre los cuales se encuentra que el $49,4 \%$ afirma haber sido víctima de atentados terroristas; $82,6 \%$, de amenazas, $21,5 \%$, de delitos contra la libertad, y $32,5 \%$, de desaparición forzada. El hecho victimizante menos frecuente en la muestra fue el haber sido víctima de minas antipersonas con un $10,9 \%$.

Tabla 2

Hechos victimizantes en los participantes

\begin{tabular}{|c|c|c|c|}
\hline & & Frecuencia & Porcentaje \\
\hline \multirow{2}{*}{$\begin{array}{l}\text { Atentados } \\
\text { terroristas }\end{array}$} & Sí & 131 & $49,4 \%$ \\
\hline & No & 134 & $50,6 \%$ \\
\hline \multirow[t]{2}{*}{ Amenaza } & Sí & 219 & $82,6 \%$ \\
\hline & No & 46 & $17,4 \%$ \\
\hline \multirow{2}{*}{$\begin{array}{l}\text { Delitos contra la } \\
\text { libertad. }\end{array}$} & Sí & 57 & $21,5 \%$ \\
\hline & No & 208 & $78,5 \%$ \\
\hline \multirow{2}{*}{$\begin{array}{l}\text { Desaparición } \\
\text { forzada }\end{array}$} & Sí & 86 & $32,5 \%$ \\
\hline & No & 179 & $67,5 \%$ \\
\hline \multirow{2}{*}{$\begin{array}{l}\text { Desplazamiento } \\
\text { forzado }\end{array}$} & Sí & 256 & $96,6 \%$ \\
\hline & No & 9 & $3,4 \%$ \\
\hline \multirow[t]{2}{*}{ Homicidio } & Sí & 81 & $30,6 \%$ \\
\hline & No & 184 & $69,4 \%$ \\
\hline \multirow[t]{2}{*}{ Masacre } & Sí & 62 & $23,4 \%$ \\
\hline & No & 203 & $76,6 \%$ \\
\hline \multirow[t]{2}{*}{ Minas } & Sí & 29 & $10,9 \%$ \\
\hline & No & 236 & $89,1 \%$ \\
\hline \multirow[t]{2}{*}{ Secuestro } & Sí & 33 & $12,5 \%$ \\
\hline & No & 232 & $87,5 \%$ \\
\hline \multirow[t]{2}{*}{ Tortura } & Sí & 49 & $18,5 \%$ \\
\hline & No & 216 & $81,5 \%$ \\
\hline \multirow{2}{*}{$\begin{array}{l}\text { Vinculación de } \\
\text { menores }\end{array}$} & Sí & 47 & $17,7 \%$ \\
\hline & No & 218 & $82,3 \%$ \\
\hline \multirow{2}{*}{ Despojo y abandono } & Sí & 200 & $75,5 \%$ \\
\hline & No & 65 & $24,5 \%$ \\
\hline
\end{tabular}

Fuente: Autores

El $37 \%$, que equivale a 98 personas, presenta un nivel bajo de problemas en la dimensión de bienestar psicológico; un $17,7 \%$ se encuentra en un nivel alto de problemas de bienestar psicológico, y el restante $45,3 \%$ está en un nivel medio en esta dimensión (ver Tabla 3). 
Tabla 3

Bienestar psicológico de los participantes

\begin{tabular}{cccccc}
\hline & & Frecuencia & Porcentaje & Porcentaje válido & Porcentaje acumulado \\
\hline \multirow{3}{*}{ Válido } & Bajo & 98 & 37,0 & 37,0 & 37,0 \\
& Medio & 120 & 45,3 & 45,3 & 82,3 \\
& Alto & 47 & 17,7 & 17,7 & 100,0 \\
& Total & 265 & 100,0 & 100,0 & \\
\hline
\end{tabular}

Fuente: Autores

El 76,6\% es decir 203 personas, presenta un nivel bajo de problemas en la dimensión de función social; el 2,3\% se encuentra en un nivel alto y el restante $21,1 \%$ se encuenta en un nivel medio de dificultades en esta dimensión (ver Tabla 4).

\section{Tabla 4}

Función social de los participantes

\begin{tabular}{cccccc}
\hline & & Frecuencia & Porcentaje & Porcentaje válido & Porcentaje acumulado \\
\hline \multirow{3}{*}{ Válido } & Bajo & 203 & 76,6 & 76,6 & 76,6 \\
& Medio & 56 & 21,1 & 21,1 & 97,7 \\
& Alto & 6 & 2,3 & 2,3 & 100,0 \\
& Total & 265 & 100,0 & 100,0 & \\
\hline
\end{tabular}

Fuente: Autores

En relación con las puntuaciones en las dimensiones del cuestionario GHQ-12, en el análisis de medias se estableció que (ver Tabla 5) las puntuaciones de la media en bienestar psicológico $(8,15)$ están por encima de las establecidas para el funcionamiento social $(4,57)$.
Al realizar el análisis de media por sexo (Tabla 6) se observa que no existen diferencias significativas entre los sexos; sin embargo, las mujeres presentan una media más alta en bienestar psicológico que los hombres.

Tabla 5

Medias de las dimensiones del GHQ-12

\begin{tabular}{cccc}
\hline Dimensión & Media & Mediana & Moda \\
\hline $\begin{array}{c}\text { Bienestar psicológico } \\
\text { Funcionamiento social y } \\
\text { afrontamiento }\end{array}$ & 8,15 & 8 & 10 \\
\hline
\end{tabular}

Fuente: Autores 
Tabla 6

Diferencias de medias por sexo en las dimensiones del GHQ-12

\begin{tabular}{|c|c|c|c|}
\hline & Sexo & Bienestar psicológico & Funcionamiento social y afrontamiento \\
\hline \multirow{3}{*}{$\begin{array}{l}\text { ఏ } \\
\text { ํㅡㄹ } \\
\text { 오 }\end{array}$} & Media & 7,28 & 4,39 \\
\hline & $\mathrm{N}$ & 74 & 74 \\
\hline & Desviación estándar & 4,431 & 3,572 \\
\hline \multirow{3}{*}{ 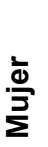 } & Media & 8,48 & 4,64 \\
\hline & $\mathrm{N}$ & 191 & 191 \\
\hline & Desviación estándar & 3,839 & 3,348 \\
\hline
\end{tabular}

Fuente: Autores

En el análisis por estado civil (Tabla 7), las personas separadas presentan la media más alta en bienestar psicológico, con un 9,29 y los divorciados las más bajas en función social, con un 4,20. En general, las medias de función social son más bajas que las de bienestar psicológico en todos los estados.

Tabla 7

Diferencias de medias por estado civil en las dimensiones del GHQ-12

\begin{tabular}{|c|c|c|c|}
\hline \multicolumn{2}{|c|}{ Estado civil } & \multirow{2}{*}{$\begin{array}{c}\text { Bienestar psicológico } \\
7,98\end{array}$} & \multirow{2}{*}{$\begin{array}{c}\begin{array}{c}\text { Funcionamiento social y } \\
\text { afrontamiento }\end{array} \\
4,39\end{array}$} \\
\hline \multirow{3}{*}{ Soltero } & Media & & \\
\hline & $\mathbf{N}$ & 92 & 92 \\
\hline & Desviación estándar & 4,206 & 3,173 \\
\hline \multirow{3}{*}{ Casado } & Media & 7,47 & 4,30 \\
\hline & $\mathbf{N}$ & 43 & 43 \\
\hline & Desviación estándar & 4,049 & 3,398 \\
\hline \multirow{3}{*}{ Divorciado } & Media & 8,60 & 4,20 \\
\hline & $\mathbf{N}$ & 5 & 5 \\
\hline & Desviación estándar & 2,702 & 1,643 \\
\hline \multirow{3}{*}{ Separado } & Media & 9,29 & 5,24 \\
\hline & $\mathbf{N}$ & 17 & 17 \\
\hline & Desviación estándar & 4,165 & 5,154 \\
\hline \multirow{4}{*}{ Viudo } & Media & 9,05 & 4,80 \\
\hline & $\mathbf{N}$ & 20 & 20 \\
\hline & Desviación estándar & 3,859 & 2,707 \\
\hline & Media & 8,20 & 4,73 \\
\hline Unión libre & $\stackrel{N}{\text { Desviación estándar }}$ & $\begin{array}{c}88 \\
3,951\end{array}$ & $\begin{array}{c}88 \\
3,509\end{array}$ \\
\hline
\end{tabular}

Fuente: Autores 
El análisis de medias por nivel educativo (Tabla 8) muestra que las puntuaciones más altas están asociadas al no tener estudios (10,33), mientras que las puntuaciones más bajas se registran en el nivel de educación técnico - tecnológico $(3,58)$.

Tabla 8

Diferencias de medidas por nivel educativo en las dimensiones del GHQ-12

\begin{tabular}{cccc}
\hline & Nivel educativo & Bienestar psicológico & $\begin{array}{c}\text { Funcionamiento social y } \\
\text { afrontamiento }\end{array}$ \\
\hline \multirow{2}{*}{ Ninguno } & Media & 10,33 & 5,93 \\
& N & 15 & 15 \\
Primaria & Desviación estándar & 3,016 & 3,693 \\
& Media & 8,70 & 5,12 \\
& N & 117 & 117 \\
Secundaria & Desviación estándar & 3,911 & 3,472 \\
& Media & 7,52 & 4,02 \\
& N & 106 & 106 \\
Técnico- & Desviación estándar & 4,057 & 3,269 \\
tecnológico & Media & 6,92 & 3,58 \\
& Desviación estándar & 24 & 24 \\
Universitario & Media & 4,272 & 3,106 \\
& N & 7,67 & 3,67 \\
& Desviación estándar & 3 & 3 \\
& & 6,028 & 2,309 \\
\hline
\end{tabular}

Fuente: Autores

\section{Discusión}

La investigación cumplió con el objetivo de describir las características de salud mental de las víctimas del conflicto armado colombiano que participaron en el estudio por medio del Cuestionario de Salud General GHQ-12.

La muestra estuvo conformada por 265 participantes, de los cuales 191 eran mujeres y 74 hombres, siendo el estado civil predominante soltero, con un $34,7 \%$, es decir, 92 personas.

Con respecto a los hechos victimizantes, los más frecuentes fueron el de víctimas de desplazamiento forzado, con un $96,6 \%$; seguido de amenazas, $82,6 \%$, y despojo y abandono con un $75,5 \%$. Los menos frecuentes fueron ser víctima de minas, 10,9\%; secuestro, 12,5\%; vinculación de menores a grupos armados, 17,7\%, y torturas, con un $18,5 \%$. Estos resultados son coherentes con los presentados por Castañeda y López (2017), donde se estableció la correlación entre salud mental y calidad de vida en salud.
Esto confirma los resultados de la Encuesta Nacional de Salud Mental realizado en Colombia en el 2015, en la cual se evidenció que el mayor hecho victimizante relacionado con el conflicto armado era el desplazamiento forzado (Ministerio de Salud y Protección Social, 2015). Sin embargo, no es posible cruzar datos entre este estudio y el realizado por los autores de la presente investigación con el fin de compararlos, ya que la encuesta realizada por el Ministerio no permite discriminar datos con la población víctima del conflicto.

Según Hewitt, Juárez, Parada, Guerrero, Romero, Salgado, \& Vargas (2016), la falta deatención psicosocial y de rehabilitación es muy alta en población víctima del conflicto; sin embargo, los resultados de este estudio muestran que, a pesar de ésto, la población no presenta puntuaciones significativas que revelen altos índices de problema psicológicas y sociales.

Lo anterior no significa que se debe dejar de trabajar en la promoción y atención en salud mental de las víctimas, sino que estas logran establecer estrategias de afrontamiento 
que les permite disminuir los riesgos para su salud.

Entre las limitaciones del presente estudio se encuentra el muestreo, que fue no probabilístico e intencional, lo que dificulta la generalización de los resultados a otras poblaciones víctimas de conflicto que presenten las mismas características de la muestra, y puede sesgar los resultados derivados de la voluntariedad de esta.

Por otra parte, debido a que la investigación no diferencia entre población clínica y noclínica, no se puedeestablecer el grado de afectación psicopatológica en la población estudiada, lo que a su vez limita el alcance clínico que pudiera establecerse derivado de este estudio. Por esta razón, en futuras investigaciones se debe tener presentes estos aspectos para discriminar otros instrumentos y muestras poblacionales que amplíen los resultados y las conclusiones que se puedan establecer al diferenciar estas dos poblaciones, y que permita ser más precisos en la obtención de puntuaciones de salud mental.

Las investigaciones futuras deben orientarse a esclarecer las diferencias entre las puntuaciones relacionadas con aspectos psicológicos de la salud mental y las relacionadas con la interacción social y las estrategias de afrontamiento. Esto permitiría implementar programas de promoción y prevención de la salud mental en población víctima del conflicto, teniendo en cuenta las dimensiones psicológicas y sociales.

Finalmente, las investigaciones comparativas entre poblaciones clínicas y no clínicas ayudarán a esclarecer las diferencias o similitudes entre estos dos grupos poblacionales y a focalizar las intervenciones teniendo como referentes las diferencias o similitudes que se deriven de los estudios.

\section{Referencias}

Castañeda, J. y López, W. (2017). Relación existente entre la salud mental y la calidad de vida en población víctima del conflicto armado interno en la Unidad de Atención y Orientación (UAO) del municipio de Soacha. (Trabajo de grado). Universidad Católica de Colombia. Recuperado de http://hdl.handle.net/10983/14046

Cortés, A., Torres, A., López, W., Pérez, C. \& Pineda, C. (2016). Comprensiones sobre el perdón y la reconciliación en el contexto del conflicto armado colombiano. Psychosocial Intervention, 25, 19-25.

Fukuda, S. (2012). The Right to Development: Reframing a New Discourse for the Twenty-First Century. Social Research, 79, 839-864.

Hewitt, N., Juárez, F., Parada, A., Guerrero, J., Romero, Y., Salgado, A. \& Vargas, M. (2016). Afectaciones psicológicas, estrategias de afrontamiento y niveles de resiliencia de adultos expuestos al conflicto armado en Colombia. Revista Colombiana de Psicología, 25(1), 125-140.

Jaramillo, E. \& Restrepo, D. (2012). Concepciones de salud mental en el campo de la salud pública. Revista Facultad Nacional de Salud Pública, 30, 202-211.

Jiménez, W. (2009). Salud mental en el posconflicto colombiano. Revista Criminalidad, 51 (1), 179-192.

López, W., Pineda, C., Murcia, C., Perilla, C. \& Mullet, E. (2013). Forgiving perpetrators of violence: Colombian people's position. Social Indicators Research, 114, 287-301.

Marks, D., Murray, M., Evans, B. \& Willing, C. (2008). Psicología de la salud: teoría, investigación y práctica. México: Manual Moderno.

Meeberg, G. (1993). Quality of life: A concept analysis. Journal of Advanced Nursing, 18, 32 - 38.

Moreno, E. \& Moriana, J. (2012). El tratamiento de problemas psicológicos y de salud mental en atención primaria. Salud Mental, 35(4), 315-328. Recuperado de http://www.revistasaludmental.mx/ index.php/salud_mental/article/view/1486/1484

Ministerio de Protección Social. (2015). Encuesta nacional de salud mental. Recuperado de http://www.odc.gov. co/Portals/1/publicaciones/pdf/consumo/estudios/ nacionales/CO031102015-salud_mental_tomol.pdf

Núñez, A., Tobón, S., Arias, D., Hidalgo, C., Santoyo, F., Hidalgo, A. \& Rasmussen, B. (2010). Calidad de vida, salud y factores psicológicos asociados. Perspectivas en Psicología (13), 11-32.

Oblitas, L. (2009). Psicología de la salud y calidad de vida. México: Manual Moderno.

Organización Mundial de la Salud. (2007). ¿Qué es la salud mental? Recuperado de http://www.who.int/ features/qa/62/es/

Peña, M., Martínez, O. \& Leal, L. (2013). Recuperación y divulgación de las memorias colectivas socioculturales del Departamento de Santander a través de filminutos históricos. I+ $D$ Revista de Investigaciones, 2(2), 20-28. Recuperado de https:// doi.org/10.33304/revinv.v02n2-2013002

Sen, A. (2000). El desarrollo como libertad. Gaceta Ecológica, (55), 14-20.

Sen, A. (2009). El valor de la democracia: El Viejo Topo.

Urzúa, A. \& Caqueo, A. (2012). Calidad de vida: una revisión teórica del concepto. Terapia Psicológica, 30 (1), 61-71.

Villa, I., Zuluaga, C. \& Restrepo, L. (2013). Propiedades psicométricas del Cuestionario de Salud General de Goldberg GHQ-12 en una institución hospitalaria de la ciudad de Medellín. Avances en Psicología Latinoamericana, 31 (3), 532-545. 